\title{
versants
}

\section{Mallarmé et Ponge, ou la formule}

\author{
Annick ETtLIN \\ Université de Genève
}

\begin{abstract}
Dans l'un de ses premiers articles, publié en 1926 dans un numéro spécial de la N.R.F. consacré à Stéphane Mallarmé, Francis Ponge, plutôt que de célébrer le maître de l'obscurité poétique ou de louer sa parole paradoxalement silencieuse, l'affuble au contraire d'une "massue cloutée d'expressions-fixes " et fait de lui le champion, tonitruant, du lieu commun. Y a-t-il chez le poète des Divagations, comme chez celui du Parti pris des choses, un art de la formule qui met en jeu le statut de la parole poétique et la rend à des usages communs? Après avoir souligné la fascination de Ponge pour le proverbe, et montré que Mallarmé aussi mesure très bien les pouvoirs de l'aphorisme, l'article entreprend de saisir sur quelle vision de la poésie de telles apologies du lieu commun s'adossent.
\end{abstract}

Keywords : Ponge, Mallarmé, formule, proverbe, pragmatisme

En 1926, Francis Ponge, fraîchement introduit dans le milieu littéraire par la grande porte de la N.R.F., publie dans un numéro que la revue consacre à Mallarmé un « hommage " surprenant, qui montre l'auteur des Poésies en faiseur de formules, de proverbes ou de maximes. L'article, intitulé "Notes d'un poème (sur Mallarmé) ", est l'un des premiers textes à sortir de ses tiroirs, la même année que Douze petits écrits mais bien avant le Parti pris des choses, qui paraîtra en 1942. C'est non sans impertinence que la plume du jeune inconnu, dans un volume auquel participent plusieurs poètes illustres, présente son idée, forcément provocatrice : le père de la modernité poétique serait à ses yeux le champion du lieu commun. Sa «massue cloutée d'expressions-fixes $»^{I}$ garantirait à tous coups la plus éclatante des victoires rhétoriques; plus radicalement même, on pourrait le « faire servir [...] comme proverbes » (OP I, I82) afin de favoriser notamment l'avancement des carrières, littéraires ou critiques. Or si l'association de la poésie au proverbe, cette sentence à l'usage de tous, est provocante, elle ne devrait pas étonner le lecteur de Ponge, qui la retrouve présente à toutes les étapes de son œuvre et convoquée avec insistance lorsqu'il s'agit de penser les devoirs du poète ou la fonction de la poésie.

Toujours dans les années I920, la proximité de la poésie au lieu com-

* Cet article a été rédigé avec le soutien du Fonds national suisse pour la recherche scientifique (projet 165064).

I Francis Ponge, « Notes d'un poème (sur Mallarmé) », in Proêmes, Euvres complètes, éd. Bernard Beugnot, 2 vol., Paris, Gallimard, "Bibliothèque de la Pléiade », I999-2002, t. I, p. I82. Dorénavant, les références à cette édition seront indiquées par le sigle OP (I ou II) et placées entre parenthèses dans le texte. 
mun fait l'objet chez Ponge de deux fables, qui lui permettent de souligner à son propos deux leçons. "Le Cycle des saisons ", recueilli dans Le Parti pris des choses, met en scène des arbres qui, personnifiés, " tâchent d'aboutir à une feuillaison complète de paroles ", " lancent, du moins le croient-ils, n'importe quelles paroles ", afin de " recouvrir entièrement [...] le monde de paroles variées ». Mais ils butent contre l'évidence dramatique de leur condition d'arbres :

Ils ne disent que « les arbres ». [...] Toujours la même feuille, toujours le même mode de dépliement, et la même limite, toujours des feuilles symétriques à elles-mêmes, symétriquement suspendues ! Tente encore une feuille ! - La même! Encore une autre ! La même ! (OP I, 23-24)

Rien n'arrête les arbres dans leur production infinie de lieux communs, si ce n'est un proverbe - un autre lieu commun -, qui survient soudainement et sans qu'on puisse l'attribuer à aucun locuteur : «L'on ne sort pas des arbres par des moyens d'arbres ». La " remarque » provoque un " retournement moral ", la chute des feuilles puis le « dépouillement », ou l'équivalent végétal, si l'on veut, du silence. La morale de l'histoire contredit plaisamment la formule dont elle tire son dénouement, dont elle ne conserve ainsi que la forme après avoir démenti son contenu, puisqu'il semble bien que l'unique antidote au lieu commun soit encore le lieu commun, le proverbe étant seul capable de venir à bout du flux des locutions. Première leçon, donc : c'est par la formule qu'on combat la formule et qu'on la vainc. La deuxième fable, pas moins métaphorique, n'offre aucune résistance à l'élucidation et se trouve même plus directement associée à la pratique de Ponge. Elle est donnée notamment dans « Les Écuries d'Augias », qui paraît dans Proêmes, et s'appuie sur la comparaison du poète à « des peintres qui n'auraient à leur disposition pour y tremper leurs pinceaux qu'un même immense pot où depuis la nuit des temps tous auraient eu à délayer leurs couleurs " (OP I, I92). Comme dans «Le Cycle des saisons », la fable présente un retournement surprenant. Le lecteur découvre en effet à la fin du texte que « déjà d'en avoir pris conscience l'on est à peu près sauvé ", la nature du travail ayant été modifiée et surtout rendue possible : «il ne s'agit pas de nettoyer les écuries d'Augias, mais de les peindre à fresque au moyen de leur propre purin ». Deuxième leçon : on fera bien de s'accommoder de l'omniprésence du lieu commun, et de s'en servir, ce qui requiert d'ailleurs « finesse » et " persévérance » et constitue un « travail émouvant ", à réévaluer.

Ponge n'oubliera pas ces deux leçons ${ }^{2}$, l'une amère et l'autre plus douce,

2 On retrouve notamment la seconde dans Méthodes (OP I, 677), Nioques de l'avant-printemps (OP II, 960) et Pratiques d'écriture ou l'Inachèvement perpétuel (OP II, IO48-IO49). 
les deux légèrement teintées d'humour, orchestrant un basculement vers l'inattendu et menant à chaque fois à une sorte de résignation moins pessimiste que pragmatique. La prégnance de sa réflexion sur le proverbe et la réhabilitation souvent explicite qu'il en opère dans ses propos sur la poésie me conduiront ici à souligner ce qui rapproche la formule de la parole poétique plutôt que ce qui l'en distingue'. L'intérêt que Ponge lui porte m'incitera aussi et parallèlement à jeter sur l'œuvre de Mallarmé un regard insolite, en gardant à l'esprit son portrait du poète en maître ès lieux communs.

En effet, je ne doute pas que le lecteur de cet article, quel que soit son rapport - plus ou moins informé, de première ou de seconde main - à l'œuvre de Mallarmé, soit au moins familier de quelques unes de ses formules. Il sait sans doute que «rien n'aura eu lieu que le lieu », qu' un coup de dés jamais n'abolira le hasard ${ }^{4}$, qu'il existe un " double état de la parole » ou qu'on déplore « la disparition élocutoire du poète » (OM II, 2I2 et 2II). Comme l'a relevé dans un article récent le critique Patrick Thériault, les textes de Mallarmé semblent « pré-découpés » pour la citation, comme si l'œuvre appelait formellement à ce qu'on la démantèle et la sectionne, ce dont les lecteurs du plus au moins scrupuleux ne se privent d'ailleurs guère. Or, l'article de Thériault prétend qu'ils auraient tort de s'en priver, puisqu'à la question « de savoir s'il n'y aurait pas dans le texte de Mallarmé quelque chose qui le rende plus susceptible qu'aucun autre d'être segmenté et, par là, d'être assimilé à bon ou à mauvais escient par les discours critiques ${ }^{5}$, il répond par l'affirmative. Si Thériault réussit à montrer que l'art de Mallarmé est en partie, comme celui de Ponge, un art de la formule, s'il donne quelques pistes pour comprendre comment les citations viennent à y saillir, reste encore à se demander pourquoi ce que le critique appelle les «pré-citations », ces formules

3 Je m'écarterai ainsi du travail déjà mené à la fin des années 1970 par Ian Higgins dans "Proverbial Ponge " : le critique, sans doute influencé par la défaveur générale dans laquelle se trouvait alors le lieu commun aux yeux des intellectuels, considérait que les proverbes évoqués par Ponge étaient marqués par l'astuce et l'intelligence d'un seul individu; il faisait ainsi de la «formule » une «formulation » (voir « Proverbial Ponge ", The Modern Language Review, 74:2, 1979, p. 320, et du même auteur, "Against Petrification. Ponge's "Baptême funèbre" ", The Modern Language Review, 78:4, I983, pp. 824-825). Plus récemment, Christophe Hanna a abordé les « essais formulatoires » de Ponge dans une perspective pragmatiste dont le présent article s'inspire ; les formules y sont toutefois abordées moins au sens linguistique qu'au sens «scientifique » du terme (voir « Essais formulatoires et tests objectivants », in Ponge résolument, éd. Jean-Marie Gleize, Paris, ENS Éditions, 2004, pp. 247-270). On pourra consulter enfin sur le sujet le travail de Marie-Laure Bardèche dans Francis Ponge ou la Fabrique de la répétition, Lausanne, Delachaux et Niestlé, 1999, pp. 69-I08.

4 Stéphane Mallarmé, Un coup de dés jamais n'abolira le hasard, in Euvres complètes, éd. Bertrand Marchal, 2 vol., Paris, Gallimard, « Bibliothèque de la Pléiade », t. I, pp. 363 et 384-385. Dorénavant, les références à cette édition seront indiquées par le sigle OM (I ou II) et placées entre parenthèses dans le texte.

5 Patrick Thériault, «Le Découpage citationnel comme fait d'écriture », Poétique, 176, 2014, p. 263. 
toutes prêtes à l'emploi, y sont aussi nombreuses. Pourquoi, en d'autres termes, Ponge a-t-il vu, et bien vu, qu'on peut « faire servir Mallarmé comme proverbes »? Quel pouvoir est celui de la formule ? Comment, enfin, la manière dont les poètes considèrent le lieu commun informe-t-elle leur vision de la poésie ? En répondant à ces questions, je serai amenée à souligner la remarquable proximité, si ce n'est formelle, du moins théorique, des œuvres de Mallarmé et de Ponge.

\section{Trahir le bruit par le bruit}

Si la formule est un outil, voire une arme, c'est surtout qu'elle permet de répliquer à d'autres formules potentiellement aliénantes, que Ponge désigne dans l'article sur Mallarmé comme " proverbes en fonte » ou " armes d'enferrement mutuel »(OP I, I82). Ce sont elles aussi que l'auteur de $\mathrm{La} \mathrm{Mu-}$ sique et les Lettres vise peut-être lorsqu'il évoque la « formule absolue » du matérialisme le plus strict, cette sentence - " n'est que ce qui est » (OM II, $67)^{6}$ - contre laquelle la poésie devra avant toutes choses se rebiffer. Sous la plume de Ponge, le poète « trahit le bruit par le bruit », il « cré[e] un outil antilogique » tiré du même matériau linguistique dont sont faits les propos du " gouvernement ", des « philosophes » et des «poètes-penseurs ", sa matière ciselée avec le plus grand soin lui procurant une "dureté » elle aussi empruntée aux discours de l'ordre et de la logique (OP I, I82). La formule est d'abord un arrangement, l'ordonnancement rigoureusement structuré des éléments qui la composent, une forme au sens propre. Elle prend chez Ponge la consistance d'une « œuvre-objet ${ }^{7}$ mais s'incarne déjà chez Mallarmé à travers la soumission du vers à la syntaxe, à laquelle le poète, qui se décrit lui-même comme «scrupuleusement syntaxier»(OM II, 7I5), prétend se consacrer. Il est frappant en effet que la forme poème soit destinée à emprunter celle, grammaticale, de la prose, et que ce soit à ce prix seulement qu'elle « revêt[e] une forme définitive » ou « sacrée »; stratégie qui lui permet de fait de « rabatt[re] » à elle « toute la prose » (OM II, 205). Particulièrement en temps de crise ou d'interrègne, à ces « moments où les proverbes ne suffisent plus ", il revient au poète de se faire « une haute idée [de son] pouvoir » et de fournir les armes de l'opposition : dans l'article de la N.R.F., Ponge fait du nom même de Mallarmé une formule renversante, transformant le mal- en bien-armé, convertissant la fragilité habituellement constitutive de

6 On trouve chez le poète d'autres façons encore de protester par la formule, dans « Confrontation » par exemple, ou dans sa réponse à une enquête "Sur la poésie » (OM II, 26I et 657).

7 Il est significatif à cet égard que Ponge, dans ses notes préparatoires à l'article de la N.R.F., prétende avoir trouvé chez Mallarmé justement " la possibilité d'une rhétorique par poète ", qui est à l'origine de l'idée, bientôt un idéal, de l'« œuvre-objet » (voir la notice de Bernard Beugnot dans OP I, 972). 
la parole poétique, sa subtilité ou sa délicatesse, en une formidable force, guerrière mais aussi sonore, " pour couvrir les autres voix surprenantes du hasard » (OP I, I82). En ayant recours à une méthode dont Mallarmé avait abusé, Ponge investit le nom comme une enseigne, il en fait une formule, mais pas celle qu'on attendait.

On peut aller jusqu'à affirmer que Mallarmé et Ponge confient au poète un rôle civique, voire politique. Les proverbes fournis par la poésie sont destinés à faire taire d'autres proverbes qui, chez Mallarmé, brident nos désirs de sublime et nos rêves de transcendance - comme la « formule absolue » du «n'est que ce qui est », déjà citée, les règles lexicales interdisant l'union parfaite des mots et des choses, évoquées dans «Crise de vers », ou encore, dans "Sauvegarde", les discours juridiques et politiques abusant de leur autorité pour s'imposer comme infaillibles. Chez Ponge, les proverbes soumettent l'homme à une logique singulière et le rendent sourd aux rhétoriques alternatives, qui sont pourtant aussi nombreuses qu'il existe de choses et de sujets. Empruntant leur forme, la poésie rivalise avec les lieux communs spécifiques d'autres discours. Quand Ponge affirme en effet que Mallarmé " n'est pas de ceux qui pensent mettre le silence aux paroles » (OP I, I82), il s'oppose à une formule que ses lecteurs ne peuvent manquer d'avoir en tête puisqu'elle figure dans le même numéro d'hommage de la N.R.F., dans l'article signé par Claudel, « La Catastrophe d'Igitur ». L'essai de Ponge, peu orthodoxe dans sa forme autant que dans son propos, présente avec brutalité le pendant positif d'un discours critique appelé à devenir dominant, qui tend à souligner comme Claudel le goût de Mallarmé pour le silence. Contre le «suprême Hamlet au sommet de sa tour ${ }^{8}$, Ponge dessine le portrait engagé d'un poète refusant de se taire, ce qui serait, affirme-t-il, « aussi dangereux dans cet ordre de valeurs que possible", un poète qui " parl[e] contre les paroles », d'accord de se laisser prendre avec elles « dans la honte où elles nous conduisent » (OP I, I96-197). Le texte de Ponge, composé à l'évidence d'une suite d'aphorismes, est aussi oraculaire que le discours critique auquel il s'oppose ; il l'est même de façon outrée et littéralement fait du bruit.

Se confronter au proverbe et reconnaître la place que la poésie est bien obligée de lui faire, c'est donc toujours se soucier de définir le devoir du poète, ses "raisons d'écrire » (OP I, I95-I97). C'est assurer la diffusion de la poésie dans la cité, mission qu'indique et dissimule à la fois le " ténu secret » (OM II, 324) sur lequel se fondent les Variations sur un sujet de Mallarmé. Si l'on connaît depuis quelques années, notamment par le biais des travaux de Jacques Rancière9, l'intérêt de l'auteur des Divagations pour la « foule »,

8 Paul Claudel, «La Catastrophe d'Igitur ", in Euvres en prose, éd. Jacques Petit et Charles Galpérine, Paris, Gallimard, «Bibliothèque de la Pléiade », I965, p. 5 IO.

9 Voir en particulier Jacques Rancière, Mallarmé. La politique de la sirène, Paris, Hachette, 1996. 
il vaut la peine de rappeler que Ponge avait quant à lui à cœur, dans le langage plus explicite auquel l'autorisaient une époque et des moyens d'adresse différents, de «montrer [...] que les poètes sont des gens comme les autres, comme vous exactement » (OP I, 683).

Larticle de Ponge porte encore un troisième coup aux lieux communs qui commandent déjà en 1926 le discours sur Mallarmé : après avoir changé le nom du poète en sa formule inversée (transformant le mal-armé en bien-armé), puis prononcé les aphorismes qui permettent de convertir le silence en bruit et le retrait en engagement (renversant ainsi un topos critique), il s'approprie enfin, peut-être sans le savoir, un autre proverbe cher à la postérité de Mallarmé, qui consiste en une variation sur le thème rebattu de son obscurité. Dans une formule apocryphe que transmettra Valéry, auquel on l'aurait répétée, «ce n'est point avec des idées [...] que l'on fait des vers $»^{{ }^{\circ}}$, ce n'est pas avec clarté que le poète s'exprime, son verbe mettant au défi toute logique rationnelle. Ainsi, le seul proverbe qui dans l'article de Ponge se conforme à la doxa critique est un faux proverbe, pas encore très bien diffusé et dont il tord légèrement le sens. En résistant à l'idée, à l'aide de cet « outil antilogique » (OP I, I82) que lui fournit Mallarmé, le poète conteste la loi, en quelque sorte physique, selon laquelle les mots ont tôt fait d'être prononcés qu'ils se transforment, non pas en monnaie d'échange, selon le propos bien connu de "Crise de vers", mais en idées. Ponge y revient longuement dans le discours qu'il retranscrit sous le titre de «Tentative orale ", dans Méthodes, et produit à son sujet une autre fable arboricole, non moins cruelle que celle $\mathrm{du}$ « Cycle des saisons » (OP I, 650-653).

Un arbre y fabrique des branches sur lesquelles il inscrit les idées en lesquelles il croit - franchise, lucidité, amour des arbres, par exemple. Un jour, arrive un bûcheron qui abat une branche de l'arbre, ce que l'intéressé considère comme normal, voire agréable : « quelquefois cela lui fait même du bien ». Mais un peu plus tard le bûcheron revient, muni d'une « cognée » que l'arbre " n'avait presque pas remarquée la première fois "; ce dernier " reconnaît [maintenant] dans le manche tout neuf de cette cognée le bois de la branche qu'on lui avait enlevée la première fois ". "Sale histoire ", commente Ponge, que celle qui fait d'un arbre le bois dont on fabrique les haches ; derrière la fable à laquelle il coupe court, s'abstenant d'en éclairer tout à fait la morale, se trouve la présomption que les idées peuvent se retourner contre celui qui les forge. Mieux vaut alors peut-être s'en tenir à produire des formes qui en seraient dénuées, c'est-à-dire des formules, à l'usage de tous.

Io Paul Valéry, « Poésie et pensée abstraite », in Euvres, 2 vol., éd. Jean Hytier, Paris, Gallimard, «Bibliothèque de la Pléiade », 1965, t. I, p. I324. 


\section{Le pouvoir de resservir ${ }^{\mathrm{II}}$}

Si chez Mallarmé " chaque désir d'expression [est] poussé à maximité ", si la forme du discours vise ainsi à s'approcher le plus possible de l'essence de la maxime, le néologisme de Ponge souligne par ailleurs sa remarquable efficacité : les «proverbes du gratuit » que fournit la poésie sont en effet avant tout « capable[s] de victoire dans une discussion pratique » (OP I, I82). De la poésie conçue comme forme commune, on peut « se servir », prétend Ponge, non seulement, comme c'est le cas pour Mallarmé, quand on est un littérateur encore en mal d'" attributs ", mais aussi, plus généralement, à chaque fois que la parole est en jeu, dans la vie. Plus tard, dans son long essai consacré à Malherbe, Ponge qualifiera les maximes d'« expressions vitales », de "proverbes du plus haut lieu » que le poète « chante pour vivre » (OP II, II4). On n'oubliera pas de relever à cet égard que les objets mis en vedette par le Parti pris des choses sont bien souvent des objets consommables, voués même, comme "Le Pain ", à rien d'autre qu'à devenir « objet[s] [...] de consommation » (OP I, 23), destinés, comme « Le Mollusque » et comme le coquillage auquel Ponge consacre des « Notes », à servir à d'autres que soi, ou à procurer comme "L'Huître » une "formule » que chacun aura tôt fait de confisquer pour son propre compte (OP I, 2I). Plus encore qu'à soi-même, il semble que les proverbes poétiques se laissent mettre à la disposition d'autrui - et qu'ils se laissent prendre ainsi dans des usages, plus ou moins honorables.

À plusieurs reprises, dès les années I920 mais surtout autour de la publication du Parti pris des choses dans les années 1940, Ponge indique s'être fixé pour objectif poétique de "ten[dre] au proverbe » : « on veut que cela serve plusieurs fois et, à la limite, pour tous les publics, en toutes circonstances, que cela gagne le coup quand ce sera bien placé dans une discussion » (OP I, 655), affirme-t-il par exemple à propos de l'énoncé poétique dans « Tentative orale $»^{12}$. Comme dans la fable des «Écuries d'Augias », l'enjeu est beaucoup moins métaphysique, et même moins éthique, que pragmatique. La poésie " se justifie » selon Ponge en tant qu'elle appartient au « monde concret», au «monde de tous les jours » (OP II, I35).

Un tel programme attribue à la parole du poète deux qualités, souvent d'ailleurs tournées en défauts : d'une part l'obscurité, qui se décline parfois en ambiguïté, et d'autre part la banalité. Afin d'être mieux saisie par celui qui en aura l'usage, afin surtout de pouvoir « être dite de nimporte quelle façon : hurlée, murmurée, accélérée, ralentie, affirmée, posée interrogativement, voire même [...] retournée » (OP I, 646), la formule n'exhibera pas son sens mais le dissimulera au contraire de deux manières possibles, soit en

II J'emprunte la formule au Savon de Ponge (OP II, 378).

I2 Voir aussi, par exemple, "My creative method » (OP I, 534), " Réponse à une enquête sur la diction poétique » (OP I, 646), Pour un Malherbe (OP II, 56), «Sur l'inspiration » (OP II, IO20). 
forgeant à son entour un mystère, soit en le faisant passer pour indifférent. «Langage absolu », il faut que l'énoncé poétique « signifie tout et rien », empruntant pour cela la forme de l'« énigme » ou celle de la « lapalissade », ce qui, aux yeux d'un Ponge apparemment insensible aux jugements de valeur esthétiques, semble revenir au même. À mesure que l'énoncé se fige, son contenu gagne plus de mobilité : dans l'article sur Mallarmé, c'est selon le même paradoxe que « n'importe quel hasard » peut être «élevé au caractère de la fixité », tandis que le " caprice d'expression » devient proverbe (OP I, I82). Sans nécessairement faire le deuil du sens, il s'agit de «transcend[er] [...] toute signification occasionnelle » (OP II, I92-I93), comme « un bon texte [qui] nourrit aussi la génération contradictoire » $(\mathrm{OP}$ I, 672) et « existe successivement pour plusieurs contemplateurs » (OP I, 645). Une telle réflexion sur la méthode passe ainsi par une apologie de la forme, et de la forme la plus rigoureuse qui soit, aux dépens du contenu.

À cet égard, certaines «formules frappantes " tiennent lieu de modèles, celles que Ponge puise dans les Fables de La Fontaine. Quand ce dernier avance que « la raison du plus fort est toujours la meilleure ", il faut comprendre selon Ponge que " c'est bien évidemment une constatation et non pas une règle », qu'il s'agit d' " une chose que les hommes ont coutume de dire et de faire », ou en d'autres termes d'un « lieu commun ». Il revient alors au poète de le mettre en une forme impérative, un " langage général » auquel on pourra avoir recours à chaque fois que le langage ordinaire ne suffit pas (OP II, I029). Par exemple, poursuit-il avec l'humour qui marque même ses textes les plus théoriques, quand l'injonction " passez-moi du sel » ne provoque pas la réaction escomptée, il devient nécessaire de la compléter par une formule à la fois plus radicale et plus efficace, comme « il faut autant qu'on peut obliger tout le monde ", maxime qui, jouant bel et bien le rôle qui s'y trouve désigné, " oblig[era] les hommes à [lui] céder ». Car la langue que le poète a pour mission de mettre à la disposition du tout-venant est " capable d'effets pratiques ». Sa valeur se mesure précisément à la quantité d'usages auxquels elle se prête : idéalement, elle « donn[e] lieu à des citations à propos de n'importe quelle discussion pratique », c'est-à-dire dans tous les cas. Comme la figue, elle a beau n'être qu'une " pauvre petite » ou encore une "pauvre gourde ", elle n'en tire pas moins sa valeur - non plus esthétique mais pragmatique - de ce qu'elle sert d'" argument massue » (OP II, 772 et 783), expression rappelant celle forgée pour décrire la poésie de Mallarmé.

La reprise systématique de l'adjectif « pratique », dans les exemples donnés ici et dans bien d'autres encore, est significative : en insistant sur l'inscription de la poésie dans la vie, et en particulier dans la vie quotidienne, " pour la conversation la plus terre à terre »(OP II, IO33), Ponge semble anticiper sur les propositions récentes d'une critique dite pragmatique, qu'illustrent par exemple les travaux de Marielle Macé. Si l'on accepte de le 
prendre au sérieux lorsqu'il affirme « désir[er] moins aboutir à un poème qu'à une formule » (OP I, 425), on peut considérer que Ponge propose déjà, comme Macé et comme d'autres chercheurs aujourd'hui, de « dévier le cours de la lecture vers ce qui se passe une fois le livre refermé $»^{13}$.

Et c'est bien de ce point de vue pragmatique aussi qu'on pourrait revenir à l'œuvre de Mallarmé, qui n'est de fait pas en reste lorsqu'il s'agit de fournir à son lecteur des formules à usages multiples, comme l'a encore soutenu dernièrement l'article de Thériault évoqué plus haut. Je survolerai ici trois exemples, qui mériteraient sans doute d'être plus amplement discutés. En particulier lorsqu'il destine sa parole à un public qui ne serait pas seulement constitué d'initiés, Mallarmé paraît en effet enclin à produire des citations utiles, qui contribueraient au rayonnement de la poésie ou participeraient à en établir une idéologie typiquement moderne, renforçant une doxa déjà imposée par les romantiques et ayant fait les preuves de son efficacité. Ainsi, au moment de quitter le journaliste Jules Huret auquel il vient de confier ses propos «sur l'évolution littéraire ", dans la fameuse enquête de I89I, le poète glisse in extremis une remarque qui n'a pas grand rapport avec ce qu'il vient de dire, mais fait tout son effet : « au fond, voyez-vous [termine-t-il], le monde est fait pour aboutir à un beau livre »(OM II, 702). Il est alors piquant de retrouver le même énoncé, sous une forme aménagée, au début de son essai sur «Le Livre, instrument spirituel » : il y précise que, suffisamment « sommaire ", il peut être tourné de plusieurs façons, "diversement cit[é] à [s]on éloge ou par blâme » (OM II, 224). Ce détail permet au moins de supposer que Mallarmé est conscient des usages que d'autres font de sa parole, qu'il s'inquiète peu de savoir si ces réappropriations sont ou non à son avantage et qu'il se prête enfin lui-même volontiers, pour les besoins de la captation, au jeu des reprises. Dans les années I890, alors que le poète est conscient de jouir d'une relative célébrité, qu'on le sollicite pour fournir articles et entretiens, il utilise peut-être aussi de la même manière l'association devenue commune, à la fin du siècle, entre poésie et anarchie. Dans sa réponse à l'enquête « sur l'explosion à la chambre des députés ", Mallarmé affirme en effet, dans une formule qu'on retrouve un peu partout autant chez ses lecteurs que chez ses non-lecteurs, qu' " [il] ne sai[t] pas d'autre bombe, qu'un livre » (OM II, 660). S'il est bien possible que l'analogie lui ait semblé douteuse, ce dont témoigne un passage de La Musique et les Lettres sur le même sujet (OM II, 72-73), il aura certainement eu conscience du pouvoir de détonation qu'un tel énoncé, fonctionnant dès lors comme un outil de promotion, était susceptible d'avoir. Enfin, sans doute pour mieux diffuser un idéal d'autonomie poétique que le siècle avait embrassé, Mallarmé forge dans « L'Action

I3 Marielle Macé, « Disponibilités littéraires. La lecture comme usage », Littérature, 155, 2009, p. 20. 
restreinte » une formule appelée à devenir fameuse, mais qui biaise de façon substantielle le propos qu'il tenait lui-même dans une version antérieure de l'article : si le livre « impersonnifié » " ne réclame approche de lecteur » (OM II, 2I7), dans les Divagations, au risque de trahir la première idée de Mallarmé, qui deux ans plus tôt et à la faveur d'un adverbe supplémentaire disait l'inverse $\mathrm{I}^{\mathrm{I}}$, c'est peut-être bien que la seconde formule a plus de chance que la première de circuler - ce dont la postérité a bel et bien donné confirmation. À partir de ces trois exemples rapides, confirmant que les formules sont élues et privilégiées par le poète, parfois au prix de concessions faites à ses idées, on peut supposer qu'il leur attribue dès lors quelque valeur, dont il reste à préciser la nature.

\section{«Créer un poncif, c'est le génie »}

Chez Ponge et chez Mallarmé, non seulement le lieu commun n'est pas haïssable mais il fait l'objet d'une construction poétique ambitieuse et d'un investissement théorique significatif. Bien que la formule ne jouisse certainement pas dans la pensée moderne du même prestige que le fragment, elle constitue pourtant chez ces deux auteurs un motif, de façon plus dérobée chez Mallarmé mais pas moins explicitement, comme on va le voir. Il faudrait donc à leur propos prendre au sérieux l'injonction bien connue de Baudelaire : au-delà de l'humour, parfois de l'ironie, avec lesquels Ponge et Mallarmé traitent la question de la formule poétique, il se peut bien qu'à leurs yeux « créer un poncif, [ce soit après tout] le génie $»^{15}$.

Ainsi, après avoir longuement exposé l'apologie que Ponge consacre au lieu commun, on peut si l'on est attentifs trouver glissée dans un article des Variations sur un sujet de Mallarmé, repris dans les Divagations, une même soumission à la formule, quoique plus discrète. Dans "Solitude ", qui clôt une série d'articles publiée mensuellement par le poète tout au long de l'année I895, articles à vocation à la fois théorique et pragmatique, dédiés à une défense et illustration de la place de la poésie dans la cité, l'essayiste tente de justifier d'abord son style, pour le moins obscur, puis le projet luimême, de nature démystificatrice. Puisqu'il s'agit en effet de mettre en cause le bien-fondé des discours esthétiques en reconnaissant qu'ils font souvent l'objet de malentendus, Mallarmé s'interroge dans «Solitude » sur les raisons pour lesquelles il y a cependant lieu d'« entonner ce qui ne rend de charme que tu », sachant que " quand un parleur affirme [...] une opinion

I4 Voir OM II, 325 : «Impersonnifié, un volume, assez pour qu'on s'en sépare comme auteur, ne réclame, autrement, approche de lecteur».

I5 Charles Baudelaire, Fusées, in Euvres complètes, 2 vol., éd. Claude Pichois, Paris, Gallimard, "Bibliothèque de la Pléiade », 2006-2007, t. I, p. 662. 
esthétique » il court le risque que "s'en défalque une sottise » (OM II, 259260). En d'autres termes, pourquoi vaut-il la peine de déflorer la poésie en discourant à son sujet, souvent d'ailleurs avec maladresse ? Sa réponse est édifiante : c'est « en vue d'ouvrager [...] quelque phrase, par exception, bien venue, cela toute la visée, parce qu'en dissertant de thèmes essentiels le rythme a chance de produire contours et lignes innés » $\left(\mathrm{OM}\right.$ II, 334) ${ }^{16}$. À la question de savoir pourquoi un propos sur l'art, énigmatique et souvent impertinent, mérite toutefois d'être tenu, Mallarmé répond que c'est pour produire ces «phrases » sollicitées par les journalistes et les admirateurs, peut-être aussi destinées plus généralement aux lecteurs de poésie afin qu'ils lui restent dévoués. Produits comme aléatoirement au fil de la discussion lorsque celle-ci s'attaque à des "thèmes essentiels ", par le miracle du style, les aphorismes de Mallarmé répondent bel et bien à une attente de son public. La formule, comme chez Ponge, a un usage, elle est même « toute la visée ».

En outre, le poncif est vu ici comme un ouvrage, dont la fabrication dépend d'une technique mais dont le contenu souvent hasardeux importe moins que la forme, ces « contours et lignes » qui, lorsqu'ils paraissent « innés », font de l'énoncé singulier une « phrase » bonne à être utilisée et redistribuée par tous les lecteurs, du moment qu'ils le désirent. Reprendre «Crise de vers ", par exemple, à la lumière de la déclaration de «Solitude " permet de mieux comprendre le commentaire de Pascal Durand, qui propose de considérer l'essai le plus fameux de Mallarmé, « composé de plusieurs morceaux cousus ensemble ", à des fins récapitulatives et peut-être publicitaires, comme un « réservoir à citations ${ }^{17}$ reposant pour commencer sur un cliché emprunté au siècle, celui de la « crise » dont l'histoire a été étudiée plus tard par Alain Vaillant ${ }^{18}$.

Le lieu commun est aimable, chez Mallarmé et Ponge, car il dure, pour commencer, comme la « résonnance » que le second tire de Malherbe et de son culte de la « raison » formelle; parce qu'il s'impose par la quantité et l'efficacité, ensuite, comme son "savon », " sujet dérisoire [peut-être] mais qui mousse interminablement »d'une part et finit par rendre " les mains plus pures » d'autre part (OP II, 388 et 37I) ; enfin parce qu'il séduit le plus grand nombre et se conforme ainsi au désir de plaire que Ponge exprime tardivement mais impétueusement dans son Nouveau nouveau recueil, parfois avec sérieux et d'autres fois de manière plus ambivalente (OP II, I063 et II86). N'est-ce pas avec tout autant d'ambiguïté que Mallarmé, dans ses écrits sur

I6 Je cite ici la version publiée dans la Revue blanche en I895, plus explicite que sa refonte pour la parution des Divagations deux ans plus tard.

I7 Pascal Durand, Crises. Mallarmé via Manet (de "The Impressionists and Edouard Manet " à "Crise de vers »), Leuven, Peeters, 1998, p.3.

I8 Alain Vaillant, La Crise de la littérature. Romantisme et modernité, Grenoble, ELLUG, 2005. 
les arts du spectacle, attribue au lieu commun théâtral - décidément mauvais sur le plan esthétique - le pouvoir de faire durer l'art quand celui-ci traverse une période d'interrègne (OM II, 199) ? C'est dire que les proverbes loués par les deux poètes impliquent des modes de légation et de diffusion pour le moins cyniques, mais qu'ils fonctionnent. Peu importe, dès lors, qu'on puisse tronquer les aphorismes de Mallarmé ou les comprendre de travers, puisque, comme l'affirme Ponge, c'est aux lieux communs de la critique qu'il revient d'assurer la postérité de l'œuvre ${ }^{19}$, même si la manière dont ils y parviennent est discutable.

À la question enfin de savoir pourquoi Mallarmé et Ponge tendent avec autant de détermination à la formule, on pourrait répondre comme Patrick Thériault, dans l'article déjà cité, qu'elle leur fournit une méthode imparable pour imposer leur auctorialité, voire leur autorité : l'art de la formule constituerait ainsi « une stratégie d'écriture ayant pour visée la légation institutionnelle et le rayonnement symbolique du nom de l'Auteur $»^{20}$. Quant à moi, j'aimerais défendre une autre hypothèse. S'il existe bien chez Ponge un paradoxe du lieu commun, inspiré d'ailleurs de Mallarmé, selon lequel on peut « faire une loi de [s]on particulier $»^{21}$, d'autant qu' entr[er] en société » permet justement «à quiconque de concevoir son identité personnelle » (OP II, 398), la promotion qu'assure la circulation de la formule n'est toutefois pas celle de l'auteur lui-même, mais d'une figure biaisée de celui-ci, à l'usage de lecteurs nombreux et impatients. Ainsi, la puissance du lieu commun permet moins de faire circuler la pensée de Mallarmé qu'une idée qu'on se ferait ensemble de ce qu'elle a pu être ; ce qui se propage n'appartient pas à l'auteur, mais à la « foule »; enfin, « créer un poncif » n'est pas dans ce cas un geste narcissique mais un acte engagé en faveur de la poésie, qui implique même un certain sacrifice de soi $^{22}$, comme dans le beau poème sur lequel se terminent les Proêmes de Ponge.

Dans ce poème en vers centré à nouveau sur un arbre, qui cette fois est sur le point de mourir, sa « trop sincère écorce » se détache de son tronc pour « rejoindre à $[\mathrm{s}] \mathrm{es}$ pieds celles des autres siècles ». Le poème est celui d'un deuil qui se prépare, c'est un poème dramatique dont le paroxysme est souvent cité par les critiques comme par Ponge lui-même : "Bien que de mes vertus je te crois la plus proche / Décède aux lieux communs tu es faite pour eux » (OP I, 23I). À l'inverse de ce que comprennent parfois les lecteurs,

I9 Voir Ponge, Pour un Malherbe, OP II, 7I.

20 Thériault, «Le Découpage citationnel comme fait d'écriture », art. cit., pp. $271-272$.

2I L'expression, issue d'une note préparatoire à l'article sur Mallarmé, est retranscrite dans OP I, 972.

22 En effet, l'œuvre idéale aux yeux de Ponge est celle qui « pourrai[t] ne pas être signé[e] du tout » (OP II, I6o), celle qui se compose de « formules si frappantes (autoritaires) et évidentes, qu'elles puissent se passer d'être signées » (OP II, 33). 
« décéd[er] au lieux communs » ne signifie pas qu'on y renonce, mais qu'on s'y voue, quelque douloureuse que puisse être l'opération. Non seulement le plus intime se transforme ici en le plus général, mais la transformation, qui se produit en même temps qu'un « volontaire auteur » se laisse « démasqu[er] volontiers », se place ainsi tout entière sous la responsabilité de ce dernier ; elle découle d'un choix qu'il aurait fait et de sa soumission volontaire à une double opération de dévoilement et de desquamage, l'auteur abdiquant par là deux fois sa posture d'autorité. Dans la parabole de Ponge, rejoindre le grand réservoir des lieux communs c'est aussi se laisser mourir, « exprès ». Le vers « Décède aux lieux communs tu es faite pour eux » montre en effet toute l'ambivalence du lien entre poésie et proverbe, lien à la fois désirable et funeste, soutenu délibérément mais non sans amertume. Le geste représenté ici est celui d'une offrande - comme celle de Mallarmé " offr[ant] » à qui voudra la brandir une "massue cloutée d'expressions-fixes » (OP I, I82) et aussi celui d'un renoncement, le décès de Ponge rappelant la condition du poète moderne qui « cède l'initiative aux mots » (OM II, 2II) et se met en retrait derrière la langue, derrière la poésie. L'auteur, dans les propos théoriques de Ponge et de Mallarmé, fait don aux lecteurs des lieux communs qui lui sont le plus chers, sachant que, dans la formule, l'idée meurt toujours au profit d'usages multiples et imprévisibles. À la fin, les lieux communs assurent la transmission non pas de noms, ni même d'idées, mais d'actes, ceux de lire et d'écrire. 